
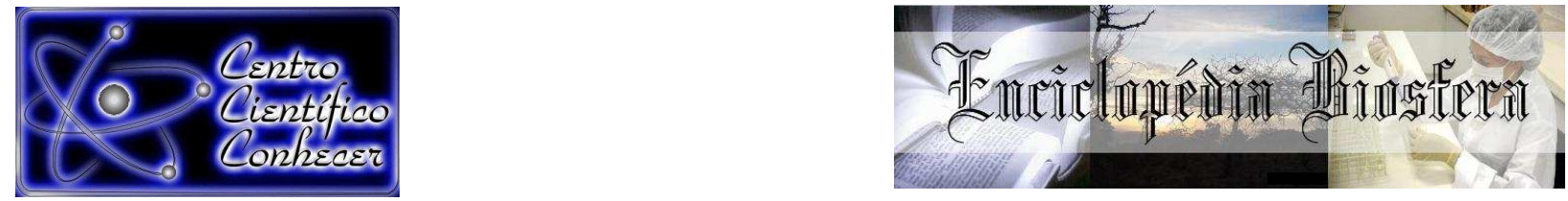

\title{
AVALIAÇÃO MACROSCÓPICA DO SISTEMA GENITAL FEMININO DO TATU- CANASTRA (Priodontes maximus - Kerr, 1792) - ESTUDO DE CASO
}

Susan Christina Braga Domingos ${ }^{1}$, Mayara Marques Pereira Fernandes ${ }^{1}$, Francisco Glauco de Araújo Santos ${ }^{2}$, Luciana dos Santos Medeiros ${ }^{2}$, Yuri Karaccas de Carvalho $^{2}$

1.Graduanda em Medicina Veterinária, Centro de Ciências Biológicas e da Natureza, Universidade Federal do Acre

2.Professor(a) Doutor(a), Centro de Ciências Biológicas e da Natureza, Universidade Federal do Acre, Rio Branco - Brasil

(ykaracas@yahoo.com.br)

Recebido em: 08/09/2015 - Aprovado em: 14/11/2015 - Publicado em: 01/12/2015

DOI: http://dx.doi.org/10.18677/Enciclopedia_Biosfera_2015_011

\section{RESUMO}

O Tatu-Canastra é um animal de hábitos noturnos e solitários, sendo considerado o maior tatu do mundo. De acordo com a IUCN (União Internacional para Conservação da Natureza), o Priodontes maximus encontra-se em situação de vulnerabilidade. Por outro lado, verifica-se que essa espécie possui ampla distribuição geográfica, sobretudo no bioma do cerrado. Diante disso, há uma notável escassez de informações acerca de suas características morfológicas, sobretudo atreladas aos aspectos reprodutivos. Esse estudo avaliou a anatomia macroscópica do sistema genital feminino de tatu-canastra. O cadáver do animal foi conservado em solução de formaldeído a $10 \%$. Posteriormente, realizou-se uma incisão na região abdominal para visualização in situ da morfologia e anatomia do sistema genital feminino. Após a visualização, o sistema genital foi retirado, dissecado, mensurado e fotografado. A fixação do sistema genital feminino do Tatu Canastra na cavidade abdominal difere da descrita para os animais domésticos. Na análise das porções do sistema genital do Priodontes maximus, pôde-se constatar semelhanças com outros animais do mesmo gênero, como a morfologia piriforme do útero e a forma ovoide dos ovários. Por outro lado, devido à raridade da espécie, novos estudos deverão ser realizados no macho da mesma espécie, a fim de elucidar e permitir intervenções reprodutivas para a preservação da espécie.

PALAVRAS-CHAVE: anatomia, morfologia, ovário, Priodontes maximus, útero.

\section{MACROSCOPIC EVALUATION OF THE GIANT ARMADILLO'S GENITAL SYSTEM (Priodontes maximus - Kerr, 1792)}

\begin{abstract}
The giant armadillo is an animal of nocturnal and solitary habits, considered the biggest armadillo in the world. According to the IUCN (International Union for Conservation of Nature), the Priodontes maximus is in a vulnerable situation. On the other hand, it appears that this species is widely spread, especially in the cerrado biome. Therefore, there is a remarkable lack of information about its morphological
\end{abstract}


characteristics, especially linked to reproductive issues. This study assessed the gross anatomy of the female genital system of the giant-armadillo. The animal's corpse was preserved in a $10 \%$ formaldehyde solution. It was done an incision in the abdominal area for viewing the in situ morphology and anatomy of the female genital system. After viewing, the genital system was removed, dissected, measured and photographed. The fixing of the female genital system of the giant armadillo in the abdominal cavity differs from that described in domestic animals. In the analysis of portions of the genital system of the giant armadillo, there were some similarities with other animals of the same genus, such as the piriformis morphology of the uterus and the ovoid shape of the ovaries. On the other hand, due to the rarity of the species, new studies should be conducted on the male of the same species, in order to elucidate and allow reproductive interventions for the preservation of the species.

KEYWORDS: anatomy, morphology, ovary, Priodontes maximus, uterus.

\section{INTRODUÇÃO}

A Superordem Xenarthra é composta por uma variedade de mamíferos, sendo eles excepcionalmente diferentes quanto à morfologia, comportamento e habitat. O Tatu Canastra, Priodontes maximus, também denominado por Tatu Gigante ou Tatu Carreta, pertencente à Superordem Xenarthra, Ordem Cingulata, é o único na Família Dasypodidae (ANACLETO \& MARINHO-FILHO, 2001; LARRÁZABAL, 2004). É considerado um animal de hábitos noturnos, sendo o maior tatu desta ordem, deste modo, pode alcançar cerca de 40 a $60 \mathrm{~kg}$. Considera-se que - Tatu Canastra é um animal de hábitos solitários, exceto nos períodos de atividade sexual (DENNIS \& MERITT, 2006).

O Tatu-Canastra encontra-se em uma variedade de habitats, ocorrendo em grande parte da América do Sul, a leste dos Andes, desde a Venezuela, Colômbia e Guianas até a Argentina, Paraguai e Brasil. Os ambientes os quais o Tatu canastra reside variam desde florestas de terras baixas e altas a savanas, sendo que o habitat de predileção deste animal consiste em áreas abertas. No Brasil é encontrado em quase todas as regiões, com exceção do nordeste, sobre o qual não há relatos (WETZEL, 1985; ANACLETO \& MARINHO-FILHO, 2001; BAUNI et al., 2013).

São considerados animais vulneráveis segundo a União Internacional para Conservação da Natureza (IUCN, 2012), com a população constantemente diminuindo. O hábito alimentar é baseado essencialmente em uma dieta de insetos, principalmente formigas e cupins, sendo mais rara a associação a outros elementos como materiais vegetais (ANACLETO \& MARINHO-FILHO, 2001). Entre as adaptações necessárias para este tipo de dieta está o formato da língua, a habilidade em escavações, evidente pelo tamanho das unhas presentes no membro torácico.

Alguns detalhes anatômicos podem caracterizar o Priodontes maximus: podem apresentar comprimento entre 75 a $100 \mathrm{~cm}$, com cauda de aproximadamente $50 \mathrm{~cm}$, e altura de cerca de $60 \mathrm{~cm}$. A porção dorsal é quase que totalmente coberta por escamas ósseas retangulares, alinhadas em fileiras. Há de 11 a 13 bandas móveis dorsais na carapaça, tornando-o extremamente flexível, e 3 a 4 bandas móveis no pescoço (SMITH, 2007). A cabeça, relativamente pequena ao corpo, é coberta por uma carapaça em formato oval, enquanto a cauda é longa, afilada e apresenta pequenas carapaças pentagonais, com a porção final desprotegida. A cabeça apresenta formato triangular. As patas são grandes e apresentam garras grandes, principalmente nos membros torácicos. 
O hábito sexual do Tatu Canastra não é totalmente conhecido, limitado pela dificuldade de acompanhamento de animais vivos e aptos à reprodução. Apesar da dificuldade de estudos, podem-se estimar certos números. Segundo NERIS et al. (2002), o período de maturidade sexual é alcançado em torno de 10 a 12 meses sendo o tempo de gestação de aproximadamente quatro meses, nascendo de 1 a 2 filhotes com cerca de $113 \mathrm{~g}$ cada. A fêmea apresenta um par de tetos que são usados para a alimentação do filhote por cerca de 4 a 6 meses.

Diante disso, motiva-se um maior conhecimento a cerca desta espécie para que sejam preenchidas algumas lacunas especialmente ao que diz respeito ao sistema genital. Devido às preocupações por conta das ameaças de extinção sofridas por este animal, objetivou-se descrever morfologicamente o sistema genital feminino do Tatu canastra, considerando aspectos macroscópicos e topográficos da espécie.

\section{MATERIAL E MÉTODOS}

O estudo foi realizado na Universidade Federal do Acre (UFAC) junto ao Laboratório de Anatomia Animal (Centro de Ciências Biológicas e da Natureza), o qual foi aprovado pelo Comitê de Ética Animal da Universidade Federal do Acre (Parecer $n^{\circ}$ 053/2015) e Sistema de Autorização e In formação em Biodiversidade SISBIO (n॰47124-1).

Para realização do presente estudo foi utilizado um cadáver de Tatu Canastra (Priodontes maximus) adulto do sexo feminino, com massa corpórea de 45 $\mathrm{Kg}$, oriundo de morte provocada por atropelamento na rodovia $\mathrm{AC}-40$, a $70 \mathrm{~km}$, próximo à cidade de Capixaba - $\mathrm{AC}$, que, inicialmente, foi resfriado e transportado ao Laboratório de Anatomia Animal na Universidade Federal do Acre.

Após fixação do cadáver por meio de solução de formaldeído a $10 \%$, realizou-se a incisão da região abdominal para abertura da cavidade. $O$ afastamento das vísceras e alças abdominais permitiu a visualização in situ do sistema genital feminino do animal. Posteriormente, realizou-se a dissecação com auxílio de uma lupa e depois com um paquímetro analógico mensuraram-se as porções do sistema. A nomenclatura utilizada para denominação das estruturas identificadas teve como base a NOMINA ANATOMICA VETERINARIA (2012).

\section{RESULTADOS E DISCUSSÃO}

O sistema genital feminino do Priodontes maximus é composto por dois ovários, duas tubas uterinas, útero simples, uma cérvix e uma vagina (Figura 1), assim como descrito em espécies como o Tatu Galinha (Dasypus novemcinctus), Tatu Mataco (Tolypeutes matacus), Tatu Peludo (Chaetophractus villosus) e Bicho Preguiça de Pescoço Marrom (Bradypus variegatus), todos pertencentes à mesma superordem Xenarthra (CETICA et al., 2005; REZENDE et al., 2010; FAVORETTO, 2014).

Após a mensuração de todos os componentes do sistema genital feminino chegaram aos seguintes resultados: ovário direito $3,42 \mathrm{~cm}$, ovário esquerdo $4,0 \mathrm{~cm}$, tuba uterina direita $5,8 \mathrm{~cm}$, tuba uterina esquerda $5,9 \mathrm{~cm}$, corpo uterino $6,6 \mathrm{~cm}$, cérvix $1,7 \mathrm{~cm}$, vagina $2,5 \mathrm{~cm}$ e vulva $3,2 \mathrm{~cm}$. 


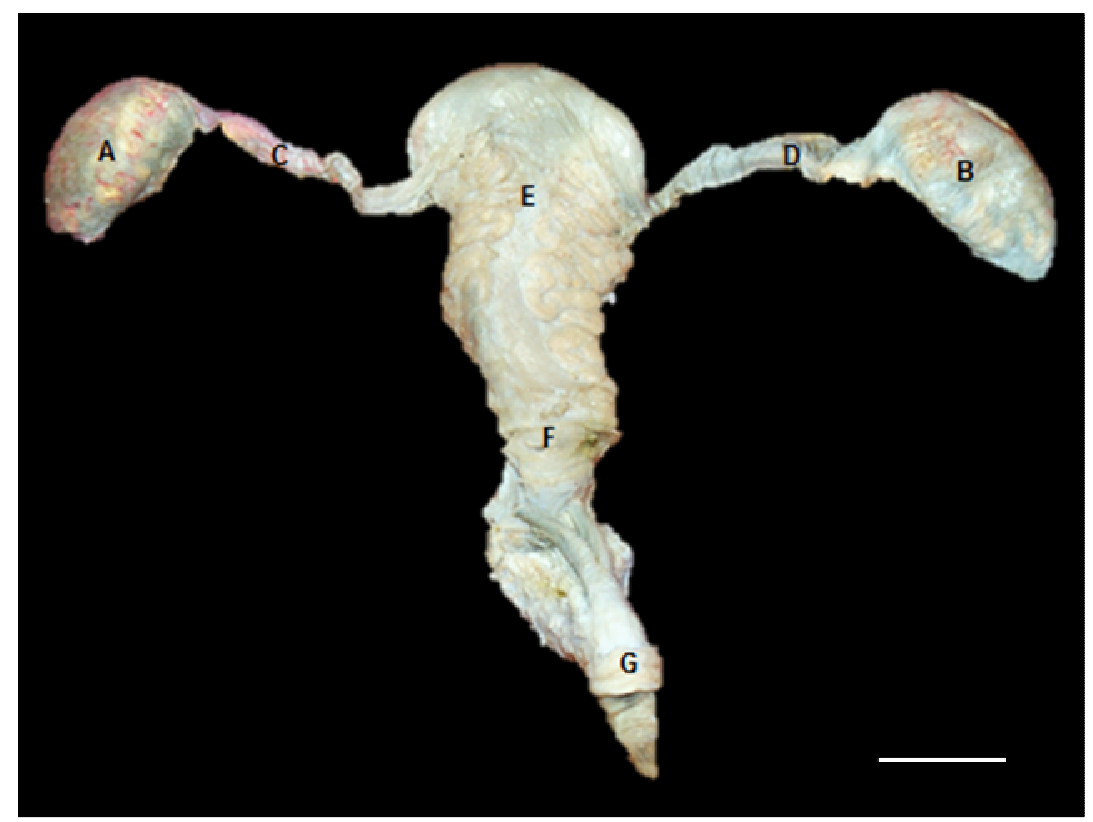

FIGURA 1. Sistema Genital Feminino do Tatu Canastra (Priodontes maximus) adulto. A. Ovário Direito; B. Ovário Esquerdo; C. Tuba Uterina Direita; D. Tuba Uterina Esquerda; E. Corpo do Útero; F. Cérvix; G. Vulva. Barra $=5 \mathrm{~cm}$. Fonte: Arquivo Pessoal (2014).

Observou-se que o sistema genital feminino do Priodontes maximus localizase na cavidade abdominal se estendendo a região pélvica, assim como o Tatu Peba (Euphractus sexcinctus) que apresenta topografia semelhante a esta observada (REZENDE, 2011). A inserção dos ovários do Tatu Canastra se dá na porção ventral-lateral da cavidade abdominal, distinguindo-se dos animais domésticos que apresentam os ovários localizados na região dorsal da cavidade abdominal (DYCE, 2010; KONIG \& LIEBICH, 2011).

De acordo com BARRETO et al. (2013), os ovários da preguiça Bradypus variegatus apresentam-se bilobados, achatados crânio-caudalmente e ovoides. Esta informação confronta-se com a morfologia observada no Priodontes maximus, que possui ovários achatados dorso-ventralmente e morfologia ovoide, bem como descrito nos tatus Chaetophractus vellerosus, Zaedyus pichi, Tolypeutes matacus e Chlamyphorus truncatus (CETICA et al., 2005; REZENDE et al., 2013).

Macroscopicamente, a tuba uterina do Priodontes maximus é um órgão de conformação tubular, filiforme e serpinginoso com calibre variado, presente nos dois antímeros, a qual projeta-se lateralmente ao útero ligando-o aos ovários. Estes achados vão de encontro aos descritos no Chaetophractus villosus, Euphractus sexcinctus e Bradypus variegatus (CODÓN \& CASANAVE, 2009; FAVORETTO, 2014).

Segundo CETICA et al. (2005) e SILVA et al. (2010), o Chaetophractus villosus e o Euphractus sexcinctus apresentam um útero bicornual, diferindo dos achados observados no Priodontes maximus, que por sua vez, apresenta um útero simples de formato piriforme achatado dorsoventralmente. Por outro lado, o padrão morfológico no Priodontes maximus é semelhante ao encontrado no útero do Tatu Mulita (Dasypus hybridus), Tatu Bola (Tolypeutes matacus), Bicho Preguiça de Garganta Marrom (Bradypus variegatus), Preguiça de Coleira (Bradypus torquatus) e Tamanduá Mirim (Tamandua tetradactyla) (CETICA et al., 2005; SILVA et al., 2010; 
FAVORETTO, 2014). Segundo REZENDE (2011), o corpo da cérvix do Tatu Peba possui um breve estreitamento nas porções dorsal e ventral, o que corrobora com os achados do presente estudo e que demonstra a proximidade de ambas as espécies.

A genitália externa do Euphractus sexcinctus, segundo REZENDE (2011), é compreendida por vulva, clitóris, abertura da vulva, uma comissura cranial e outra caudal e encontra-se no plano mediano da região perianal, com aparência de órgão único de estrutura firme e formato cônico. Dentro deste contexto, encontram-se similaridades com os achados observados no Priodontes maximus.

\section{CONCLUSÃO}

A fixação do sistema genital feminino do Tatu Canastra difere da descrita nos animais domésticos. Na análise das porções do sistema genital do Priodontes maximus pôde-se constatar semelhanças com outros animais do mesmo gênero. Nesse viés, destaca-se a morfologia piriforme do útero e a forma ovoide dos ovários. Por outro lado, devido à raridade da espécie, novos estudos deverão ser realizados no macho da mesma espécie, a fim de elucidar e permitir intervenções reprodutivas para a preservação da espécie.

\section{AGRADECIMENTOS}

À Universidade Federal do Acre por possibilitar o desenvolvimento deste estudo e pela concessão da bolsa de Iniciação Científica.

\section{REFERÊNCIAS}

ANACLETO, T. C.; MARINHO-FILHO, J. Hábito alimentar do tatu-canastra (Xenarthra, Dasypodidae). Revista Brasileira de Zoologia, v. 18, n. 3, p. 681-688, 2001.

BARRETO, M.LM.; AMORIM, M.J.A.A.L.; FALCÃO, M.V.D. Análise Morfológica e Morfométrica das Gônadas de Preguiça (Bradypus variegatus Schinz, 1825). Pesquisa Veterinária Brasileira, v. 33, n. 9, p.1130-1136, 2013.

BAUNI, V.; CAPMOURTERES, V.; HOMBERG, M.A.; ZULETA, G.A. Distribution and Status of the Extant Xenarthrans (Mammalia: Xenarthra) in the Southern cone Mesopotamian Savanna, Argentina. Edentata, v.14, p. 35-50, 2013.

CETICA, P.D; MERANI, M.S; MARCOS, H.J.A. Morphology of female genital tracts in Dasypodidae (Xenarthra, Mammalia): a comparative survey. Zoomorphology, v.124, n. 2, p.57-65, 2005.

CODÓN, S. M; CASANAVE, E. B. Morphology and Histological Annual Changes of the Oviduct of Chaetophractus villosus (Mammalia, Xenarthra, Dasypodidae). International Journal Morphology, v. 27, n. 2, p. 355-360, 2009.

DENNIS, A.; MERITT, JR. Research Questions on the Behavior and Ecology of the Giant Armadillo (Priodontes maximus). Edentata, v.7, p. 30-33, 2006.

DYCE, K.M.; WENSING, C.J.G.; SACK, W.O. Tratado de anatomia veterinária. 4 ed. Rio de Janeiro: Elsevier, 2010. 856p. 
FAVORETTO, S.M. Estudo Morfológico do Sistema Reprodutor Feminino do Bicho-Preguiça-de-Garganta-Marrom (Bradypus variegatus, SCHINZ, 1825). 2014. 34 f. Tese (Mestrado) - Faculdade de Ciências Agrárias e Veterinárias da Universidade Estadual Paulista, Universidade Estadual Paulista, São Paulo. 2014.

INTERNATIONAL COMITEE ON VETERINARY GROSS ANATOMICAL NOMENCLATURE. Nomina Anatomica Veterinaria. 5. ed. Hannover: Sapporo, 2012. $160 \mathrm{p}$.

IUCN. IUCN Red List Categories and Criteria: Version 3.1. 2 ed. Gland, Switzerland and Cambridge: International Union for Conservation of Nature, 2012, 32p.

KONIG, H.E.; LIEBICH, H.G. Anatomia dos Animais Domésticos: Texto e Atlas Colorido. 4 ed . Porto Alegre: Artmed, 2011. 788p.

LARRÁZABAL, L. B. Crianza en cautiverio de perezoso de dos dedos (Choloepus didactylus). Edentata, v. 6, p. 30-36, 2004.

NERIS, N.; COLMAN, F.; OVELAR, E.; SUKIGARA, N.; ISHII, N. Guía de mamíferos medianos y grandes del Paraguay: distribución, tendencia poblacional y utilización. Asunción: SEAM, 2002. 165p.

REZENDE, L.C.; SILVA, M.P.; ALCÂNTARA, D.; MIGLINO, M.A. História, Biogeografia e Análise Filogenética dos Xenarthras (Mammalia). Enciclopédia Biosfera, v. 6, n. 10, 8 p, 2010.

REZENDE, L.C. Biologia da Reprodução em Tatus: Análise Morfológica do Aparelho Reprodutor Feminino da Espécie Euphractus sexcinctus e Análise Morfológica Placentária Comparativa entre as Espécies Chaetophractus villosus, Chaetophractus vellerosus e Euphractus sexcinctus. 2011. 143 f. Tese (Doutorado) - Faculdade de Medicina Veterinária e Zootecnia, Universidade de São Paulo, São Paulo. 2011.

REZENDE, L.C.; KÜCKELHAUSB, S.A.S.; GALDOS-RIVEROSA, A.C.; FERREIRA J.R.; MIGLINOA, M.A. Vascularización, morfología e histología del ovario en el armadillo Euphractus sexcinctus (Linnaeus, 1758). Archivos de Medicina Veterinaria, v. 45, n. 2, p.191-196, 2013.

SILVA, M.P.; REZENDE, L.C.; ALCÂNTARA, D.; MIGLINO, M.A. Análise Comparativa da Morfologia Uterina do Bicho-Preguiça, Tamanduá e Tatu (Xenarthras). Enciclopédia Biosfera, v. 6, n. 10, 7 p, 2010.

SMITH, P. GIANT ARMADILLO (Priodontes maximus). Mammals of Paraguay, n. 6, 2007.

WETZEL, R. M. The taxonomy and distribution of armadillos, Dasypodidae. In: MONTGOMERY, G. G. The Evolution and ecology of sloths, armadillos, and vermilinguas. Washington: De Smithsonian, 1985. 451p. 